\title{
Rol de los egresados de la Facultad de Odontología de la Universidad Nacional Mayor de San Marcos en Instituciones Odontológicas
}

Performance of graduates ones of the Faculty of Dentistry of Universidad Nacional Mayor de San Marcos in Dentistry Institutions

\section{Resumen}

El egresado de odontologia en su desempeño profesional, realiza labores no solamente en el ámbito exclusivo del tratamiento de las enfermedades orales, también, como portador de la identidad profesional, lidera los procesos de transformación de la sociedad y del desarrollo de la profesión, ejerciendo cargos en Instituciones Educativas Universitarias, en Instituciones Odontológicas profesionales científicas y gremiales. Los modelos que acreditan carreras universitarias reconocen al egresado y su desempeño social como factor importante para la emisión del Juicio de Calidad de la carrera en mención. El objetivo de este trabajo es evidenciar la presencia de los egresados de odontología de la Universidad Nacional Mayor de San Marcos en las instituciones profesionales a través del tiempo, y motivar a los miembros de la comunidad odontológica sanmarquina sobre el rol a desempeñar en el desarrollo de la Odontología Peruana, analizando el rol de los egresados sanmarquinos de odontología que participan liderando instituciones gremiales y científicas de nuestro país. El estudio realizado es descriptivo, transversal a nivel exploratorio. Se recolectó información de los archivos de las instituciones mediante una ficha. La muestra fue no probabilística intencional. Los resultados confirman que los egresados, en los últimos 20 ańos han venido liderando las diferentes Instituciones Odontológicas con una participación de 65\%, en las instituciones odontológicas nacionales.

Palabras clave: egresados, calidad, desempeño.

\begin{abstract}
Professional performance of graduates in dentistry, is not only exclusive in the area of oral diseases treatment, but, as a bearer of professional identity, he leads the process of societal transformation and the development of the profession, exercising positions in university educational institutions, in scientific and professional dental institutions unions. Models that accredit university carriers recognize the graduate bachelor and his social performance as an important factor for the issue of quality judgments of the carrier in mention. The aim of this paper is to demonstrate the presence of graduates of dentistry from Universidad Nacional Mayor de San Marcos in professional institutions through time, and motivate members of San Marcos dental community about the role to play in the development of Peruvian Dentistry, analyzing the role of San Marcos dental graduates in leading scientific institutions of our country. The study is descriptive, transversal and exploratory. Information was collected from the archives of institutions through a card. The sampling was not ramdom. The results confirm that graduates in the last 20 years have been leading various institutions with a 55\% stake in national dental institutions. Key words: graduated, quality, performance.
\end{abstract}

\section{Introducción}

Los estudios de seguimiento de egresados son una herramienta importante para analizar los caminos que siguen los nuevos profesionales, no solo en lo que respecta a su inserción laboral, sino al entorno y contexto en que se desenvuelven. Son también mecanismos poderosos de diagnóstico de la realidad con el potencial de inducir en las instituciones la reflexión sobre sus fines y valores, desde que aportan elementos para analizar las ventajas y desventajas de los diferentes modelos y opciones educativas que permitirán su fortalecimiento o revisión". ${ }^{1}$
En las últimas décadas se ha convertido en una necesidad creciente de las instituciones educativas, la incorporación de líneas de investigación que permitan un análisis riguroso sobre aspectos de su vida institucional, para tomar decisiones y establecer programas bien estructurados en lo curricular y lo presupuestario, condición sin la cual estarían en desventaja al afrontar retos y demandas de una sociedad cada vez más participativa y exigente. En ese marco el seguimiento de egresados junto a otras líneas de investigación retroalimentan la pertinencia curricular y sus posibles adecuaciones, dentro de una congruencia regional y nacional. El seguimiento de
Carlos Arroyo Pérez ${ }^{1}$, Nidia

L. Fernández Diez', Jaime

A. Sánchez García', Carlos Villafana Mori ${ }^{1}$, Patricia B. Astupinaro Capristan', Saúl Ilizarbe Escajadillo ${ }^{1}$

\footnotetext{
Dpto. Académico de Estomatología Rehabilitadora. Facultad de Odontología. Universidad Nacional Mayor de San Marcos.
}

\section{Correspondencia:}

Mg. Carlos Arroyo Pérez

Av. Germán Amezaga s/n, Ciudad Universitaria

Facultad de Odontología UNMSM.

Teléf.: 993-982-362

E-mail: carlosarroyo_56@hotmail.com

Fecha de recepción: 27-04-10

Fecha de aceptación: 24-03-11

egresados como línea de investigación no sólo tiene relación con el análisis y reformulación curricular, sino que tiene además, una importante articulación con la inserción en el mercado laboral de los egresados. ${ }^{2}$

En la Facultad de Odontologia la UNMSM, no existe un estudio previo, con respecto al tema, no obstante en países como México, Argentina, Espańa, Colombia, Venezuela y países Europeos, se han visto motivados para realizar estudios de seguimiento a sus egresados y así poder establecer cuál es el rol de estos frente a la sociedad en sus diversos aspectos, mas ninguno hace 
mención al protagonismo institucional que estos pudieran tener en el ámbito de gestión.

En Argentina, por ejemplo, los resultados de un estudio permitieron inferir la importancia de la evaluación y seguimiento de los egresados, así como reforzar los planes y programas de estudio para anticipar y proponer adecuaciones detectando demandas futuras y potenciando la función innovadora y crítica de la universidad. ${ }^{3}$

El proceso de autoevaluación contempla como parte de uno de sus factores, el desempeño de sus egresados, conocer dónde están ubicados, qué están haciendo, cuáles son sus vínculos con la universidad que los formó y cómo los vincula la universidad al enriquecimiento de sus programas. ${ }^{4,5}$

La idea de que el trabajo del odontólogo es una cuestión simple debe evolucionar, pues sus alcances profesionales pueden trascender más allá de lo que de manera tradicional se conoce. Según Botero, estos profesionales pueden trabajar en el área de diseño, bien sea de prótesis o de equipos para facilitar el trabajo. La docencia, investigación, creación de empresa, administración y auditoría son otros campos de acción para el odontólogo. ${ }^{6}$

Las instituciones de educación superior deben brindar una mayor atención a sus egresados para poder adaptar sus proyectos profesionales a la demanda profesional en cuanto a las competencias que requiere el mercado laboral. ${ }^{7}$

Los estudios de egresados, desde un enfoque sistémico, permiten la evaluación de la universidad o programa como un todo. Se evalúan los insumos o condiciones de funcionamiento, los procesos, los productos y resultados. La evaluación del producto de las universidades (sus egresados), además de ser el propósito principal de los estudios de egresados, también puede tener un efecto de realimentación a través del cual se pueden evaluar las necesidades de los egresados de la propia universidad bajo estudio para mejorar su formación. Por otro lado, puede propiciar una mejor comunicación entre la institución y los egresados. $^{8}$

Es importante tener en cuenta que realizar estudios de opinión de empleadores en educación no es una tarea sencilla, pues requiere inversión, tiempo y un trabajo bien organizado, que al final de cuentas nutrirá los procesos académicos, ya que dará frutos al ver a los profesionales insertarse en empleos acordes con su formación profesional. Para ello, es necesario investigar y promover opciones pedagógicas y curriculares que contribuyan a vincular la universidad con las fuerzas sociales; así los egresados encontrarán un entorno social adecuado para desenvolverse. ${ }^{9}$

La Escuela Normal Superior Oficial Guanajuato ha desarrollado algunos estudios de egresados, cuyos objetivos se han centrado en conocer la opinión de estos en torno a la pertinencia, utilidad y relevancia de los contenidos estudiados durante su formación normalista. Sin embargo, no hay estudios de seguimiento de egresados enfocados al desempeńo profesional, ni en la detección de competencias derivadas de su formación profesional en la Escuela Normal, lo cual implica que no se tenga información sobre su desempeño real a partir de haber cursado un Programa de Estudios que propone un perfil de egreso inespecífico. ${ }^{10}$

Por otro lado, situando a los egresados bajo la perspectiva de un capital humano que mediante la relación que existe entre educación y trabajo termina por reconocerse que el empleo es una meta natural y de perspectiva siempre presente en la educación, donde finalmente el egresado se suma a una actividad social y que tendríamos que considerar que en el campo profesional el aprendizaje es visto como participación social, de integración a una comunidad de práctica. Significa entonces que estas comunidades refinan sus propias prácticas y garantizan nuevas generaciones de miembros ${ }^{10}$.

Las universidades siempre han considerado el seguimiento de egresados como una labor significativa. La Universidad Autónoma Metropolitana (UAM) y la Asociación Nacional de Universidades e Instituciones de Educación Superior (ANUIES) han sido promotoras permanentes de este tipo de estudios. La UAM publicó en 1997 un trabajo dirigido por Giovana Valenti sobre los egresados de la UAM en el mercado de trabajo. La ANUIES también ha hecho lo propio y en 1996 publicó "Diferenciación institucional de la educación superior y mercados de trabajo", elaborado por Carlos Muñoz Izquierdo. Sin embargo, es al arribo de Julio Rubio a la Secretaría General de la ANUIES cuando se da un paso decisivo en cuestión de seguimiento de egresados con la publicación del "Esquema básico para estudios de egresados", coordinado por Magdalena Fresán y publicado en 1998, convirtiéndose rápidamente en una referencia metodológica obligada para entender el tema y su evolución en nuestro sistema de educación superior. ${ }^{11}$

Los trabajos realizados a la fecha no contemplan el aspecto protagónico que desempeñan los profesionales Odontologos como líderes en su comunidad, ocupando cargos directivos, en diferentes Instituciones Odontológicas y es lo que motiva realizar la presente investigación.

\section{Material y método}

Es un estudio descriptivo, a nivel exploratorio. Para este trabajo se consideró como población a los máximos representantes de las instituciones Académicas y Gremiales tanto de Lima como de provincias (Presidentes de la Federación Odontológica del Perú, Presidentes de la Sociedad Peruana de Prótesis Dental y Maxilofacial, presidentes de la Academia de Estomatología, y Presidentes de la Asociación Odontológica del Ministerio de Salud (MINSA). La unidad muestral fue conformada por los Decanos y Presidentes de las instituciones que ejercieron dichos cargos en los últimos 20 ańos. El tipo de muestreo fue no probabilístico e intencionado. Para la recolección de datos se usó el método retrospectivo, recolectándolos de los archivos y registros de las Instituciones. Los datos obtenidos fueron consignados en una ficha ad-hoc, confeccionada y validada para los propósitos de la investigación. Para la recolección de datos se contó, aparte del responsable de la investigación, con cinco integrantes del equipo de estudio. Después se procedio a realizar un proceso orientado a uniformizar criterios respecto a la estructura de la ficha. Hecho esto, se efectuó una prueba de procedimiento, con el fin de determinar la eficiencia de la ficha, realizándose las modificaciones y ajustes del caso. La ficha fue remitida a las instituciones mencionadas adjuntando un oficio en el que se detalló los propósitos del trabajo. La información recabada fue centralizada y procesada para el análisis final de los datos y presentación de resultados.

\section{Resultados}

Los resultados nos demuestran que la Facultad de Odontología de la Universidad Nacional Mayor de San Marcos mantiene un fuerte liderazgo en las diferentes instituciones consultadas. Así, tenemos que 55 egresados de Odontología sanmarquinos han ocupado cargos directivos en 
las cuatro instituciones investigadas, lo que corresponde al $65 \%$, de los cargos ocupados en las Instituciones odontológicas gremiales y cientificas estudiadas. Se pudo constatar también que en la Academia de Estomatología del Perú, San Marcos tiene un 95\% de participación, en la Sociedad de Protesis dental y Maxilo Facial tambien San Marcos esta liderando con una participación del $70 \%$. Mientras que en la Federación Odontologica del Perú la que lidera es la Universidad San Luis Gonzaga con una participación del 52 $\%$, mientras que San Marcos tiene una participación correspondiente al $43 \%$.

\section{Discusión}

En la Academia de Estomatología, son dos las universidades que han aportado integrantes a la plana directiva, la Universidad Nacional Mayor de de San Marcos destaca con un $95 \%$ de participación y la Universidad Peruana Cayetano Heredia con un $5 \%$.

Así mismo, en la Sociedad de Prótesis Dental y Máxilo Facial, se destaca la participación de la Universidad Nacional Mayor de San Marcos en la actividad dirigencial con un $70 \%$, mientras que la Universidad Nacional Federico Villarreal, Cayetano Heredia y San Luis Gonzaga de Ica, tienen un 10\% de participación cada una.

Igualmente, en la Asociación de Odontólogos del MINSA destacan los egresados de odontología de la Universidad Nacional Mayor de San Marcos con un $52 \%$, seguido por la Universidad Federico Villarreal con $29 \%$, y la Universidad San Luis Gonzaga de Ica con 19 \%,

Tabla 1. Cargos Directivos en Instituciones Odontológicas desempeñados por egresados de Odontología según Universidad de origen

\begin{tabular}{|c|c|c|c|c|c|c|c|c|c|c|}
\hline \multirow[t]{2}{*}{ Universidad } & \multicolumn{2}{|c|}{$\begin{array}{c}\text { Federación } \\
\text { Odontológica } \\
\text { del Perú }\end{array}$} & \multicolumn{2}{|c|}{$\begin{array}{c}\text { Sociedad de } \\
\text { Prótesis Dental } \\
\text { y Máxilo Facial }\end{array}$} & \multicolumn{2}{|c|}{$\begin{array}{l}\text { Academia } \\
\text { de Estoma- } \\
\text { tología }\end{array}$} & \multicolumn{2}{|c|}{$\begin{array}{c}\text { Asociación } \\
\text { de Odon- } \\
\text { tólogos } \\
\text { MINSA }\end{array}$} & \multicolumn{2}{|r|}{ Total } \\
\hline & $\mathrm{n}$ & $\%$ & $\mathrm{n}$ & $\%$ & $\mathrm{n}$ & $\%$ & $\mathrm{n}$ & $\%$ & $\mathrm{n}$ & $\%$ \\
\hline UNMSM & 9 & 43 & 15 & 70 & 20 & 95 & 11 & 52 & 55 & 65 \\
\hline UNFV & 1 & 5 & 2 & 10 & 0 & 0 & 6 & 29 & 9 & 11 \\
\hline UNICA & 11 & 52 & 2 & 10 & 0 & 0 & 4 & 19 & 7 & 20 \\
\hline $\mathrm{UPCH}$ & 0 & 0 & 2 & 10 & 1 & 5 & 0 & 0 & 3 & 4 \\
\hline Total & 21 & 100 & 21 & 100 & 21 & 100 & 21 & 100 & 84 & 100 \\
\hline
\end{tabular}

Universidad Nacional Mayor de San Marcos (UNMSM), Universidad Nacional San Luis Gonzaga de Ica (UNICA), Universidad Federico Villarreal (UNFV) y Universidad Peruana Cayetano Heredia (UPCH) de lo cual vale destacar que desde el año 2003, la Universidad Nacional Mayor de San Marcos mantiene el liderazgo en esta Institución.

Sin embargo, podemos apreciar que en la Federación Odontológica del Perú, la Universidad San Luis Gonzaga de Ica ha logrado tener el liderazgo desde el año 2000 hasta el 2009 en forma consecutiva, seguido por la Universidad Nacional Mayor de San Marcos y la universidad Federico Villarreal ocupando el tercer lugar.

$\mathrm{Al}$ hacer la proyección global de la participación de los egresados de odontología en las instituciones odontológicas podemos destacar que los egresados de la Facultad de Odontología de la Universidad Nacional Mayor de San Marcos tienen el liderazgo con un $65 \%$ de participación, seguidos por los egresados de odontología de la Universidad Nacional San Luis Gonzaga de Ica con un $20 \%$, la Universidad Nacional Federico Villarreal con un 11\%, y la Universidad Peruana Cayetano Heredia con un $4 \%$.

\section{Conclusiones}

Este trabajo confirma que la Facultad de Odontología de la Universidad $\mathrm{Na}$ cional Mayor de San Marcos mantiene un liderazgo en la mayoría de las Instituciones Odontológicas que se han investigado. Esto nos compromete a reflexionar acerca de los determinantes en la formación de nuestros estudiantes en liderazgo analizando si nuestro currículo contempla cursos que fomenten a este.

\section{Referencias bibliográficas}

1. Lezcano D, Coscarelli N, Irigoyen S, Albarracín S, Medina M, Mosconi E, Rueda L, Papel G, Seara S, Tomas L. Sistema de Evaluación y seguimiento de los egresados. Universidad de La Plata, Facultad de Odontología; 2010 [actualizado marzo 2011; citado 04 de abril de 2011]. Disponible en: http:// es.scribd.com/doc/40117528/F2Lezcano

2. Medina M, Mosconi E, Albarracín S; Papel G, Coscarelli N, Rueda L, Irigoyen S. Modelo para evaluación y seguimiento de los egresados aplicado en la Facultad de Odontología de la UNLP. VII Coloquio internacional sobre gestión universitaria en América del sur. Nov. a Dic. del 2007. Disponible en: http://www. inpeau.ufsc.br/wp-content/BD_ documentos/2007.

3. Albarracín S, Coscarelli N, Mosconi E, Papel G, Rueda L, Medina M, Irigoyen S. Perfil de los Egresados de la F.O.L.P. entre el 01-01- 1998 al 31-12-2001.Facultad de Odontología - Universidad Nacional de La Plata - cosmoruepa@sinectis. com.ar Acceso:[25 de Abril del 2009]. Disponible en: http://rapes. unsl.edu.ar/Congresos_realizados/ III\%20Encuentro/Completos/ALBARRACIN.pdf

4. Alvarado ME. "El seguimiento de egresados de estudios profesionales". México, D.F. Ed. del CISE_ UNAM Series sobre la Universidad. Acceso [25 de Abril del 2009].

Disponible en: http://olimpo.reduaz.mx/sip/doctos_grales/CIEESOdontologia/CIEESODONTOLO GIA/CARPETA\%20 NUEVE/50\%20SEGUIMIENTO\%20DE\%20EGRESADOS. pdf. http://www.ens.edu.mx/pdf/ Eje 3,7_Tema 6pdf.files/F2\%20 Mosconi.pdf

5. Omaña CO. "Seguimiento de egresados bachillerato en Hidalgo México". Acceso: [26 de Abril del 2009]. Disponible en: http://tecnologiaedu.us.es/edutec/paginas/35.html.

6. Ospina VN. "Odontología ahora en la Autónoma de las Américas," 3 de Octubre del 2008. El Colombiano. com. Acceso: [26 de Abril del 2009]. Disponible en: http://www.elcolombiano.com/BancoConocimiento/O/ odontologia_ahora_en_la_autonoma_de_las_americas/odontologia_ 
ahora_en_la_autonoma_de_las_ americas.asp.

7. Argote L A, Duque A M, O.D., González L, Payán A, Payán C, Rojas L, Tovar M. Articulo - DOC. EGRESADOS. Los egresados y su desempeño en el medio: un desafío de las instituciones formadoras del recurso humano en salud. Acceso: [3 de Mayo del 2009] Disponible en: http://www.inpeau.ufsc.br/coloquio03/Completos/ALBARRACIN.doc.

8. Green I, Vargas A, Gonzales R, Ruiz R, Fruto O, Escobar V et al. Estudio de seguimiento de egre- sados de programas de Posgrado Regionales Centroamericanos. Acceso: [2 de Mayo 2009]. Disponible en: http://daad.csuca.org/ attachments/121MEMORIA\%20 ESTUDIt

9. Arellano RM. Estudio de opinión de empleadores Acceso: 5 de Mayo del 2009. Disponible en: www.ujat. $\mathrm{mx} /$.../Programaegresadosempleadores.pdf. Disponible en: http:// www.ucol.mx/acerca/coordinaciones/cgd/dgesn/files/estest/Empleadores.pdf

10. Changoyan G P. Seguimiento de los egresados una mirada al desempeño profesional, Escuela Normal Oficial de Guanajuato. Ponencia en: Encuentro Nacional de Educación Normal. Acceso: [28 de Abril del 2009]. Disponible en: http://www. ens.edu.mx/pdf/Eje3,7Tema6.pdf. Enero del 2008, pp. 1-13.

11. Sánchez CA. Hacia un Sistema Nacional de seguimiento de Egresados. Suplemento Universitario Campus Milenium 310, Jueves 26 de Febrero del 2009. Acceso [10 de Mayo del 2009]. Disponible en: http://www.campusmilenio.com. $\mathrm{mx} / 310 / . . . /$ egresados.php 